\title{
MENTAL HEALTH STATUS AND HELP-SEEKING STRATEGIES OF INTERNATIONAL STUDENTS IN CANADA
}

\author{
\begin{tabular}{c|c|c} 
DANELLE DE MOISSAC & JAN MARIE GRAHAM & KEVIN PRADA \\
UNIVERSTIÉ DE SAINT-BONFACE & BRANDON UNIVERSITY & UNIVERSTTÉ DE SAINT-BONFACE
\end{tabular} \\ NDEYE ROKHAYA GUEYE \\ UNIVERSTTÉ DE SAINT-BONFACE \\ RHÉA ROCQUE \\ UNIVERSITY OF WINNIPEG
}

\begin{abstract}
International students are at heightened risk of developing psychological distress, yet little research has been conducted on their mental health or support needs. This quantitative study focused on undergraduate students at two mid-sized universities in Manitoba, Canada. Online and paper surveys were completed by 932 participants, of whom $21 \%$ identified as international students. This paper, descriptive in nature, outlines the sociodemographic profiles, current mental health status, psychological characteristics, and coping strategies of international students compared to domestic students in each institution. Data show that international students are more likely to report excellent mental health, score higher on the mental health scale, and report higher life satisfaction, higher self-esteem, and more positive body image than domestic respondents. However, they are less likely to talk about their hardships. Providing culturally-adapted supports that take into consideration ethnolinguistic differences, religious practice, and mental health literacy will better meet the needs of international students on campus.
\end{abstract}

Keywords: international students, undergraduate, mental health, support services, ethnolinguistic diversity

\section{Résumé}

Les étudiants internationaux sont à risque de détresse psychologique; or, peu de recherches portent sur leur santé mentale et leur besoin de soutien. Cette étude quantitative a été menée auprès d'étudiants de premier cycle dans deux universités du Manitoba, au Canada. Un sondage a été rempli par 932 participants, dont $21 \%$ qui s'identifiaient comme étudiants internationaux. De nature descriptive, l'article décrit et compare le profil sociodémographique, l'état de santé mentale, les caractéristiques psychologiques et les stratégies d'adaptation des étudiants internationaux et canadiens dans chaque établissement. Selon nos données, les étudiants internationaux rapportent plus souvent avoir une excellente santé mentale, être satisfaits de leur vie, avoir une bonne estime de soi et une image corporelle positive que les étudiants canadiens. Toutefois, ils sont moins enclins à parler de leurs problèmes. Un soutien adapté aux différentes cultures qui prendrait en considération les différences ethnolinguistiques, les pratiques religieuses et la littératie en santé mentale répondrait mieux aux besoins des étudiants internationaux.

Mots-clés : étudiants internationaux, premier cycle, santé mentale, services de soutien, diversité ethnolinguistique

\section{Introduction}

Since the turn of the millennium, globalization has transformed the landscape of higher education in Canada: internationalization of Canadian post-secondary campuses is flourishing (Canadian Bureau for International
Education [CBIE], 2018a). In the 2017-2018 academic year, the number of international students (IS) in Canada-originating from over 200 countries-increased for the third successive year, representing $14.1 \%$ of overall enrolment and a $376.5 \%$ increase since 2000 (Statistics Canada, 2018a, 2020). Program and policy 
developments aimed specifically at attracting students seeking an education outside their home country (Statistics Canada, 2018b), the reputable quality of Canadian education, and the perception of a safe, tolerant, and non-discriminatory society may further account for this proliferation (CBIE, 2018b). For their part, IS enrich the Canadian post-secondary landscape, providing meaningful opportunities for multilateral dialogue in various intellectual and cultural spheres, thus enriching all students' knowledge of global history and issues. These exchanges may, in turn, assist emerging adults seeking to develop cross-cultural competencies, invaluable in our globalized world (Guo \& Guo, 2017; Lokkesmoe et al., 2016).

\section{Post-secondary Students' Mental Health}

Mental health is a pressing issue in North American higher education; post-secondary students are at greater risk of experiencing psychological distress than the general population (Acharya et al., 2018; Cleary et al., 2011; Kovess-Masfety et al., 2016; Kruisselbrink Flatt, 2013). This vulnerability may be triggered by the convergence of several developmental challenges experienced by emerging adults (Arnett, 2005) as they begin their post-secondary journey. Challenges include academic, work, housing and financial responsibilities, coupled with the potential for risky lifestyle choices amid newfound liberties and pressures (Kruisselbrink Flatt, 2013). These factors may have a marked effect on students' psychological well-being (Gloria \& Steinhardt, 2016), adaptability (Nelson \& Padilla-Walker, 2013), and psychosocial functioning (Dawson \& Pooley, 2013; Farrell \& Langrehr, 2017), which may negatively impact academic performance and success (American College Health Association, 2019; Owens et al., 2012). To counter declining student mental health, on-campus counselling is increasingly provided, and has been shown to have a positive impact on student grades and overall well-being (Schwitzer et al., 2018).

\section{International Students' Mental Health}

IS are at even greater risk of exhibiting mental health distress than their domestic counterparts (Clough et al., 2019; Forbes-Mewett \& Sawyer, 2016; Prieto-Welch, 2016; Shadowen et al., 2019). Additional obstacles faced by IS include student living (lodging, nutrition), social and spiritual needs, finances, academic pressure (including language development), and transitioning into the Canadian society and post-secondary context (Calder et al., 2016; Smith et al., 2013). They may also face anxiety resulting from the unknown, strained personal relationships, loneliness, isolation (Mesidor \& Sly, 2016; Prieto-Welch, 2016), and discrimination (Shadowen et al., 2019). Various factors may impede IS' recourse to mental health services. These include preference for self-reliance, or personal or collective perceptions and beliefs surrounding mental health, often referred to as mental health literacy (MHL), which is essential to help-seeking behaviour development (Beks et al., 2019; Rafal et al., 2018). For others, poor awareness of health care service availability (Onabule \& Boes, 2013; Robinson et al., 2016) and cultural stigma surrounding the pursuit of, and commitment to, mental health treatment (Beks et al., 2019; Eisenberg et al., 2009) are greater obstacles. These challenges may lead to disparate use of and, consequently, limited benefit from mental health services.

\section{Acculturation and Language Barriers}

Upon entering their host country, IS engage in a process of acculturation-a period of dynamic cultural and psychological change resulting from contact between members of two or more cultural groups (Berry, 2005). This acculturative process may encompass alterations in an individual's beliefs, values, identity, or behaviour (Abraído-Lanza et al., 2004; Thomson \& Hoffman-Goetz, 2009). Although of value for personal and professional enrichment, this process may lead to acculturative stress, which has been associated with poor mental health, internalizing symptomology, and increased risk behaviour (Crockett et al., 2007; Huang \& Mussap, 2018; Yang et al., 2018), especially among undergraduates, females, mature students, students residing with family, and students who have resided in their host country for two years or more (Kim et al., 2019).

Limited English-language proficiency may further intensify acculturative stress (Fritz et al., 2008; Prieto-Welch, 2016; Shadowen et al., 2019), as it can hinder the ability of IS to effectively communicate and seek help (Prieto-Welch, 2016), consequently leading to mental health troubles (Brisset et al., 2014; Kirmayer et al., 2011). Language barriers may also impede participation in class lectures, assignment quality, and creation 
of positive social connections with domestic students (Shadowen et al., 2019). As suggested in the literature, language and culture are key factors to consider when tailoring support services to such a heterogeneous group (Martirosyan et al., 2019; Prieto-Welch, 2016; Robertson et al., 2015).

\section{Support Services for International Students}

In the two top worldwide study destinations for IS-the United States and the United Kingdom (CBIE, 2018b)_services ${ }^{1}$ designed to support IS' social integration, academic achievement, and language development are provided (Bartram \& Terano, 2011; Martirosyan et al., 2019). According to a Canadian study describing IS' experiences in Ontario universities, culturally-congruent support services $^{2}$ should focus on health and well-being, and offer access to on-campus physical and mental health services (Smith et al., 2013). With Canadian post-secondary campuses increasingly becoming culturally and linguistically diverse, institutions recognize that supports promoting retention, engagement, and academic success should be better suited to the needs of IS (Anderson, 2015; Guo \& Guo, 2017).

\section{Objectives}

Few studies have focused on IS mental health and access to on-campus support services on Canadian campuses, despite international literature highlighting the significant difficulties faced by this student population (Clough et al., 2019; Shadowen et al., 2019). With Canada being the fourth leading international study destination globally (CBIE, 2018b), gaining insight into IS' well-being and mental health needs is critical for student retention and success. Canada's bilingual identity makes it a particularly attractive study destination for those coming from English and French-language countries; ethnolinguistic differences impacting mental health and access to mental health services by IS in Canada should be explored.

This article reports on a subset of a broader quantitative study investigating post-secondary student mental health and risk-taking behaviours among five Canadian universities (de Moissac et al., 2019). It focuses on a cohort analysis of participants from one French-language and one English-language university in Manitoba, Can- ada, comparing domestic students' and IS' sociodemographic and ethnolinguistic profiles, mental health status and psychological characteristics, as well as commonly employed coping strategies and supports. Associations between groups (domestic and IS in both universities) in relation to psychological and mental health characteristics, adjusted for specific sociodemographic variables, are explored. Findings provide valuable insight into IS' needs, informing educators, administrators, and policy makers seeking to promote overall wellness among this student population.

\section{Study Context}

Although Manitoba had the second-lowest proportion of IS among mainland provinces in 2018 (CBIE, 2018a), $14.1 \%$ of its post-secondary enrolments were international, with $58 \%$ coming from Asia and $24 \%$ from Africa (Statistics Canada, 2018a). International newcomers who select Manitoba as their destination do so for its lower cost of living, moderate taxation, affordable housing, and well-established immigrant population (Economic Development Brandon, 2020; Economic Development Winnipeg, 2020; Province of Manitoba, 2020).

Both universities selected for this study are located in Manitoban cities where English is the majority language. Table 1 provides a comparison of Winnipeg and Brandon population sociodemographic and linguistic profiles. Although few differences are noted, Brandon is a smaller city with a lower proportion of French-speakers and speakers of a non-official language.

Université de Saint-Boniface (USB), a French-language institution located in Winnipeg, is Western Canada's largest Francophone university. Approximately 1,400 students are enrolled, primarily in undergraduate or college programs; $16 \%$ of enrolments are international, mostly from French-speaking African countries such as Senegal, Morocco, and the Democratic Republic of Congo. IS benefit from tailored support services ${ }^{3}$ promoting their integration through the on-campus International Office, as well as student accessibility services and academic advisors available to all students. Since 2018, part-time counselling services are available free of charge to all students via a community-based agency, offered in French or English, either on-campus or at the agency. IS may further access mental health services via their private medical insurance plan. Brandon University (BU), an English-language establishment located in 
Table 1

Winnipeg and Brandon sociodemographic and ethnolinguistic profiles (Statistics Canada, 2017)

\begin{tabular}{lcc}
\hline \multicolumn{1}{c}{ Variable } & Winnipeg & Brandon \\
\hline Total population $(\mathrm{n})$ & 705,244 & 58,003 \\
Population speaking French, $\mathrm{n}(\%)$ & $71,225(10.22)$ & $3,260(5.72)$ \\
$\begin{array}{l}\text { Population reporting a non-official language as their mother } \\
\text { tongue, } \mathrm{n}(\%)\end{array}$ & $179,355(25.76)$ & $8,930(15.65)$ \\
$\begin{array}{l}\text { Proportion reporting having earned less than \$30,000 in 2015 (\%) } \\
\text { Median total household income (\$) }\end{array}$ & 41.32 & 38.37 \\
$\begin{array}{l}\text { Prevalence of low-income population based on the low-income } \\
\text { measure, after tax, among adults 64 and younger (\%) }\end{array}$ & 68,402 & 69,656 \\
Non-Canadian citizens, $\mathrm{n}(\%)$ & $80,550(11.67)$ & 12.5 \\
\hline
\end{tabular}

the City of Brandon, in southwestern Manitoba, offers undergraduate, graduate, and certificate programs to over 3,600 students. IS represent $11.5 \%$ of the student population and come primarily from Nigeria, China, Jamaica, Ghana, and India. The on-campus International Office provides student advocacy, including referrals to two full-time mental health counsellors and academic advisors, who are accessible to all students. At the time of this study, all students had access to the I.M.Well App (Integrated Mental Wellness) through their student health insurance provider.

\section{Methodology}

\section{Design and Procedure}

A quantitative approach was used to measure socio-economic profiles, mental health indicators and psychological characteristics. Students attending undergraduate programs were invited to participate in an online or paper-based survey distributed on campus, with the possibility of completing it during class time. The survey was administered by research assistants, who also obtained informed consent from participants. The study was approved by the Research Ethics Boards of both universities. ${ }^{4}$

\section{Sample}

An invitation was sent to all students by email from the student association, providing a link to the survey. Students from pre-selected classes and classes in which faculty members were in agreement to provide time for survey administration were invited to participate; this was done to ensure adequate representation from all programs and years of study. Data collection was conducted in the Fall/Winter terms of 2018-2019. A total of 932 domestic (lived in Manitoba prior to post-secondary studies) and international (emigrated from another country to enroll in post-secondary studies in Manitoba) students participated; 639 were from USB and 293 from $\mathrm{BU}$. Of these, 172 were IS from USB, 38 from BU.

\section{Measures}

The 66-question survey, developed by the research team (de Moissac et al., 2019), focused on academic and socio-economic status, ethnolinguistic identity, mental health and associated behaviours, substance use, sexual practices, road safety, and use of new technologies. Survey question terminology was verified among international and domestic students to ensure accessibility of vocabulary employed. Data pertaining to mental health (indicators, psychological characteristics and coping strategies) are presented here, as well as demographic, ethnolinguistic and socio-economic data including 
age, gender, academic enrolment, living arrangements, financial stability, origin, language most commonly used at home, religious affiliation and practice, and ethnolinguistic identity.

Questions pertaining to mental health included the Mental Health Continuum-Short Form (Keyes, 2002, 2009), which allows the classification of mental health into three categories (flourishing, moderate, or languishing), taking into consideration emotional, social, and psychological well-being. Other mental health indicators, such as suicidal ideation, depression, anxiety, and sleep deprivation, as well as self-reported general physical and mental health, were measured, as were psychological characteristics (life satisfaction, self-esteem, and positive body image). Self-esteem was measured using the single-item scale, as it has psychometric properties equivalent to Rosenberg's Self-Esteem Scale (Brailovskaia \& Margraf, 2018). Self-esteem and life satisfaction were measured using a 5-point Likert scale; body image was measured by four category responses. Due to the small BU IS sample size, responses for some variables were combined into four categories or less.

The Multigroup Ethnic Identity Measure (Phinney, 1992), modified by Roberts and collaborators (1999) and validated by Ponterotto and collaborators (2003), was employed to measure ethnolinguistic identity. This questionnaire consists of twelve questions divided into two categories: self-affirmation and belonging, such as a feeling of pride and well-being related to ethnic identity, and exploration of and engagement with this identity. A total score was calculated by averaging scores of all items; higher scores indicate a stronger sense of belonging.

The survey was made available online through LimeSurvey, and in print. Data from hard copies were transferred to the LimeSurvey platform.

\section{Statistical Analysis}

A post hoc power analysis using G*Power 3.1.9.2 with two-sided and $Z$ test for multivariate logistic regression analysis was used to determine the power of the present study. Based on odds ratios of Group variable (Domestic vs. IS) as the main predictor (OR varied from 0.8 to 3.4 ), and adjusting for the effect of other covariates (Nagelkerke $R^{2}$ range 0.02 to 0.22 ), the total sample size of 932 students demonstrated an observed power greater than $90 \%$, except for the loss of sleep variable $(70 \%)$.
For each outcome variable (mental health indicators and psychological characteristics), students with missing values regarding the outcome variable were excluded. Missing responses were taken into consideration as a categorical dummy variable to determine if the likelihood of missing data were reflective of other differences among students. No significant differences were found between missing and no-missing values on all sociodemographic variables.

Sociodemographic variables, mental health indicators, and psychological characteristics were described as proportions for categorical variables, and as means and standard deviations (SD) for continuous variables. Chi-square or Fisher's exact tests and Anova were used to identify statistically significant differences between four groups of students (domestic USB, international USB, domestic BU, and international $\mathrm{BU}$ ), with respect to categorical and continuous variables. Due to small BU IS sample size, Fisher's exact tests were used especially when more than $20 \%$ of cells had expected frequencies $<5$.

Several multivariate binary or ordinal logistic regression analyses, with respect to dichotomous or ordinal responses, were performed to compare mental health and psychological characteristics of the two student groups (domestic and international) and institutions (USB and $\mathrm{BU}$ ) in the same model; adjustment for confounding sociodemographic variables was limited to age and gender due to the small sample size of BU IS. Adjusted odds ratio and $95 \%$ confidence interval were also reported. Goodness of fit was assessed by the Hosmer-Lemeshow multivariate binary logistic regression model and by Deviance and Pearson statistics for multivariate ordinal regression model.

All statistical analyses were performed using SPSS version 24.0 for Windows (SPSS, Inc., Chicago, IL) and G*Power (Franz Paul, Kiel, Germany). Level of significance was set at $p<.05$.

\section{Results}

Results are presented for domestic and international students for each institution separately, as cohort profiles differ significantly. 


\section{Table 2}

Sociodemographic profiles comparing domestic and international students, by university

\begin{tabular}{|c|c|c|c|c|c|c|}
\hline \multirow[b]{2}{*}{ Variable } & \multicolumn{2}{|c|}{ Université de Saint-Boniface } & \multicolumn{2}{|c|}{ Brandon University } & \multirow[b]{2}{*}{$\begin{array}{l}\text { Observed } \\
\text { statistic }^{1}\end{array}$} & \multirow[b]{2}{*}{$p$ value } \\
\hline & $\begin{array}{c}\text { Domestic } \\
\text { students } \\
n=467\end{array}$ & $\begin{array}{c}\text { International } \\
\text { students } \\
n=172\end{array}$ & $\begin{array}{c}\text { Domestic } \\
\text { students } \\
n=255\end{array}$ & $\begin{array}{c}\text { International } \\
\text { students } \\
n=38\end{array}$ & & \\
\hline Age in years: mean (s.d. $)^{2, a, c, d, f}$ & $21.2(5.0)$ & $23.9(6.1)$ & $22.1(5.45)$ & $24.7(6.26)$ & 12.9 & $0.000^{*}$ \\
\hline Female $^{3}$ & 79.8 & 42.7 & 78.6 & 54.1 & 96.89 & $0.000^{*}$ \\
\hline Spent most of life in urban centre & 73.6 & 93.0 & 47.1 & 76.3 & 104.66 & $0.000^{*}$ \\
\hline First-year student & 35.0 & 45.8 & 34.0 & 39.5 & 7.34 & 0.062 \\
\hline Full-time student & 90.5 & 94.8 & 92.9 & 100 & 6.89 & 0.075 \\
\hline \multicolumn{7}{|l|}{ Past-year academic average } \\
\hline Below $60 \%$ & 3.2 & 3.0 & 7.1 & 8.1 & & \\
\hline $60-100 \%$ & 94.2 & 87.6 & 88.2 & 73.0 & 36.09 & $0.001^{*}$ \\
\hline Not studying last year & 2.6 & 9.5 & 4.7 & 18.9 & & \\
\hline \multicolumn{7}{|l|}{ Living with } \\
\hline Alone & 2.8 & 18.6 & 10.2 & 21.1 & & \\
\hline Partner & 9.4 & 13.4 & 21.2 & 21.1 & & \\
\hline Family (with parents) & 79.0 & 8.7 & 31.4 & 2.6 & 403.09 & $0.000^{*}$ \\
\hline Family (without parents) & 3.4 & 23.8 & 6.7 & 5.3 & & \\
\hline Friends & 4.9 & 32.0 & 29.0 & 50.0 & & \\
\hline Married or common law & 14.6 & 21.2 & 20.0 & 26.3 & 7.42 & 0.060 \\
\hline Have dependent children & 6.0 & 15.3 & 8.2 & 10.8 & 14.13 & $0.003^{*}$ \\
\hline \multicolumn{7}{|l|}{ Hours worked/week } \\
\hline None & 21.7 & 48.5 & 36.2 & 28.9 & & \\
\hline Fewer than 20 hours & 62.9 & 30.4 & 47.2 & 55.3 & 61.75 & $0.000^{*}$ \\
\hline 20 hours or more & 15.5 & 21.1 & 16.5 & 15.8 & & \\
\hline \multicolumn{7}{|l|}{ Annual personal income } \\
\hline$\$ 0$ & 5.9 & 30.7 & 8.7 & 24.3 & & \\
\hline$\$ 1-\$ 15,000$ & 77.5 & 45.2 & 72.2 & 62.2 & 93.14 & $0.000^{*}$ \\
\hline$\$ 15,000$ and more & 16.6 & 24.1 & 19.0 & 13.5 & & \\
\hline
\end{tabular}




\begin{tabular}{lcccccc}
\hline & \multicolumn{2}{c}{ Université de Saint-Boniface } & \multicolumn{2}{c}{ Brandon University } & \\
\cline { 2 - 5 } Variable & Domestic & International & Domestic & International & Observed & $p$ value \\
& students & students & students & students & statistic $^{1}$ & \\
& $n=467$ & $n=172$ & $n=255$ & $n=38$ & \\
\hline
\end{tabular}

Estimated personal debt (\%)

\begin{tabular}{|c|c|c|c|c|c|c|}
\hline$\$ 0$ & 57.3 & 71.7 & 48.6 & 67.6 & \multirow{3}{*}{28.55} & \multirow{3}{*}{$0.001^{*}$} \\
\hline$\$ 1-\$ 15,000$ & 28.3 & 13.9 & 31.2 & 26.5 & & \\
\hline$\$ 15,000$ and more & 14.4 & 14.5 & 20.2 & 5.9 & & \\
\hline Financially dependent on parents & 57.5 & 67.3 & 43.9 & 59.5 & 24.43 & $0.001^{*}$ \\
\hline Have not volunteered (last year) & 29.8 & 46.7 & 36.5 & 37.8 & 100.10 & $0.000^{*}$ \\
\hline \multicolumn{7}{|l|}{ Language most often used at home } \\
\hline English & 63.5 & 9.5 & 94.5 & 57.9 & \multirow{4}{*}{547.26} & \multirow{4}{*}{$0.000^{*}$} \\
\hline French & 15.5 & 70.4 & 0 & 0 & & \\
\hline English and French & 18.6 & 4.7 & 0 & 0 & & \\
\hline Other & 2.4 & 15.4 & 5.5 & 42.1 & & \\
\hline \multicolumn{7}{|l|}{ Religious affiliation } \\
\hline Christian (including Catholic) & 55.1 & 35.9 & 27.9 & 54.0 & \multirow{4}{*}{106.62} & \multirow{4}{*}{$0.000^{*}$} \\
\hline Muslim & 2.5 & 58.7 & 0.8 & 5.4 & & \\
\hline Agnostic or Atheist & 28.0 & 3.6 & 14.2 & 10.8 & & \\
\hline Other ${ }^{4}$ & 14.1 & 1.8 & 32.8 & 29.7 & & \\
\hline Regular religious practice & 44.2 & 86.3 & 41.0 & 70.3 & 385.39 & $0.000^{*}$ \\
\hline \multicolumn{7}{|l|}{ Ethnolinguistic score mean (s.d.) ${ }^{2}$} \\
\hline $\begin{array}{l}\text { Affirmation and sense of belonging } \\
c, e, f\end{array}$ & $3.03(0.61)$ & $3.1(0.62)$ & $2.94(0.65)$ & $3.5(0.72)$ & 9.32 & $0.000^{*}$ \\
\hline Exploration and engagement $\mathrm{b}, \mathrm{e}, \mathrm{f}$ & $2.68(0.67)$ & $2.74(0.63)$ & $2.40(0.68)$ & $2.82(0.78)$ & 12.92 & $0.000^{*}$ \\
\hline Global score ${ }^{b, c, e, f}$ & $2.88(0.6)$ & $2.95(0.58)$ & $2.72(0.6)$ & $3.22(0.69)$ & 10.72 & $0.000^{*}$ \\
\hline
\end{tabular}

Notes: Data are reported in percentages; $p$ value calculated using Chi-square or Exact tests except for age and ethnolinguistic score.

1: $\chi^{2}$ de Pearson or F statistic.

2: $p$ value calculated using Anova test.

3: Few participants reported gender identification as "other," hence this category was not included.

4: Including Indigenous or other spiritualities without religious affiliation.

Pairwise comparison for Anova test: a: Domestic USB vs. International USB; b: Domestic USB vs. Domestic BU; c: Domestic USB vs. International BU; d: International USB vs. Domestic BU; e: International USB vs. International BU; f: Domestic BU vs. International BU.

${ }^{*}$ Statistical significance at $5.0 \%$.

\section{Sociodemographic and Ethnolinguistic Profiles}

Comparison of socio-economic and ethnolinguistic pro- files of the four student groups (Table 2) reveals statistically significant differences for all variables except for being a first-year student, a full-time student, and married or living common-law. 
Compared to their domestic counterparts, IS in both institutions were generally older, more often male, from urban centres, and not studying in the previous year. Most participants reported having earned an academic average above $60 \%$ in the previous academic year. Most lived with friends ( $32.0 \%$ at USB, $50.0 \%$ at BU), although USB IS were also more likely to reside with family members as compared to BU IS, who were more likely to live either alone or with their partner. Although more IS reported earning no annual income, a greater proportion of them reported relying financially on their parents $(67.3 \%$ at USB, $59.5 \%$ at BU) and having accrued no student debt (71.7\% at USB, $67.6 \%$ at BU), compared to domestic counterparts. More IS at USB reported not working (48.5\%), not volunteering in the previous year (46.7\%), and having dependent children (15.3\%) as compared to other groups. However, those who were employed were more likely to work more than 20 hours per week and to earn more than $\$ 15,000$ annually.

IS at USB were predominantly from Africa (88\%), although some were from Europe (7\%) or the Americas (5\%). A majority (70.4\%) spoke the institution's teaching language (French) most often at home, compared to $15.5 \%$ of USB domestic students; few spoke English at home (14.2\%), as compared to $57.9 \%$ of international respondents at BU. BU IS hailed from Africa (40\%), Asia (34\%), the Americas (23\%), or Europe (3\%); $42.1 \%$ spoke a language other than English at home. With respect to religious affiliation, IS at USB were more likely to be Muslim (58.7\%) or Christian (35.9\%), whereas BU IS were more often Christian (54.0\%) or of another affiliation $(29.7 \%)$. IS in both universities were more likely to report regular religious practice, with a higher percentage among USB IS (86.3\%). IS at BU scored significantly higher than other groups for affirmation and sense of belonging score, exploration and engagement score, and global ethnolinguistic identity score.

\section{Mental Health Profile}

Mental health indicators, scores, and psychological characteristics are presented in Table 3. In both universities, more IS self-reported excellent or very good general health ( $66.7 \%$ at USB, $60.5 \%$ at BU), and excellent or very good mental health ( $62.8 \%$ at USB, $60.5 \%$ at BU) compared to their domestic counterparts. Likewise, both international cohorts were more likely to obtain higher mental health scores than domestic respondents: $68.2 \%$ at USB and $70.0 \%$ at BU scored as exhibiting flourishing mental health. IS from both universities scored significantly higher in psychological well-being and average total mental health scores than domestic students. The gap between international and domestic students is greater at USB, where IS scored significantly higher in all three mental health dimensions (emotional, social, and psychological well-being) and in average total mental health score than domestic students.

With respect to mental health indicators, IS in both universities reported having planned or attempted suicide at a similar degree to local students. IS at USB were more likely to report depression, using a laxative or exercising excessively to control their weight, and were less likely to report suicidal thoughts and anxiety than other groups. IS at BU were less likely to report loss of sleep because of worries than other groups.

Concerning psychological characteristics, most IS from both institutions reported positive self-esteem (80.5\% at USB, $68.4 \%$ at BU), and obtained higher average self-esteem scores. When asked about their body image, a greater proportion of IS reported being always satisfied with their body image than their domestic counterparts.

Select mental health indicators and psychological characteristics were further analyzed to adjust sample for age and gender. As shown in Table 4, IS were 3.44 and 1.95 times more likely to report good mental health and score with flourishing mental health as compared to domestic students. However, they were also 1.6 times more likely to report depression, but less likely to report anxiety. Psychological characteristics, including life satisfaction, self-esteem, and positive body image, were $1.54,2.63$, and 2.11 times more likely, respectively, to be reported by IS than domestic students. Of note, significant differences are observed between universities: overall, USB students are more likely to present flourishing mental health and report depression and loss of sleep than BU students, who were more likely to report anxiety. 


\section{Table 3}

Mental health indicators and psychological characteristics of domestic and international students, by university

\begin{tabular}{|c|c|c|c|c|c|c|}
\hline \multirow[b]{2}{*}{ Variable } & \multicolumn{2}{|c|}{ Université de Saint-Boniface } & \multicolumn{2}{|c|}{ Brandon University } & \multirow[b]{2}{*}{$\begin{array}{l}\text { Observed } \\
\text { statistic }^{1}\end{array}$} & \multirow[b]{2}{*}{$p$ value } \\
\hline & $\begin{array}{c}\text { Domestic } \\
\text { students } \\
n=467\end{array}$ & $\begin{array}{l}\text { International } \\
\text { students } \\
n=172 \\
\end{array}$ & $\begin{array}{c}\text { Domestic } \\
\text { students } \\
n=255 \\
\end{array}$ & $\begin{array}{c}\text { International } \\
\text { students } \\
n=38\end{array}$ & & \\
\hline \multicolumn{7}{|c|}{ Mental health indicators } \\
\hline \multicolumn{7}{|l|}{ Self-rated health (\%) } \\
\hline Excellent/Very good & 53.6 & 66.7 & 53.4 & 60.5 & & \\
\hline Good & 32.0 & 27.3 & 32.0 & 34.2 & 14.491 & $0.025^{*}$ \\
\hline Fair/Poor & 14.3 & 6.1 & 14.6 & 5.3 & & \\
\hline \multicolumn{7}{|l|}{$\begin{array}{l}\text { Self-rated mental or emotional } \\
\text { health (\%) }\end{array}$} \\
\hline Excellent/Very good & 26.3 & 62.8 & 27.8 & 60.5 & & \\
\hline Good & 33.3 & 22.6 & 34.1 & 26.3 & 92.565 & $0.000^{*}$ \\
\hline Fair/Poor & 40.4 & 14.5 & 38.1 & 13.2 & & \\
\hline \multicolumn{7}{|l|}{ Mental health categories (\%) } \\
\hline Flourishing & 52.2 & 68.2 & 41.5 & 70.0 & & \\
\hline Moderate & 39.7 & 27.2 & 49.3 & 23.3 & 29.511 & $0.000^{*}$ \\
\hline Languishing & 8.1 & 4.6 & 9.2 & 6.7 & & \\
\hline \multicolumn{7}{|l|}{ Mental health score (mean) ${ }^{2}$} \\
\hline Emotional well-being ${ }^{a, c}$ & 10.53 & 11.53 & 10.42 & 10.38 & 5.16 & $0.002^{*}$ \\
\hline Social well-being ${ }^{a, b, d}$ & 13.83 & 15.85 & 12.01 & 13.76 & 13.15 & $0.000^{*}$ \\
\hline Psychological well-being ${ }^{a, d, f}$ & 20.26 & 22.85 & 18.98 & 22.14 & 11.31 & $0.000^{*}$ \\
\hline Totala $a, c, d$ & 44.61 & 50.16 & 41.5 & 46.06 & 10.98 & $0.000^{*}$ \\
\hline Depression (last year, \%) & 44.2 & 51.9 & 35.1 & 36.8 & 12.46 & $0.006^{*}$ \\
\hline \multicolumn{7}{|l|}{ Suicidal ideation (last year, \%) } \\
\hline Serious thoughts & 16.0 & 7.4 & 15.1 & 13.2 & 7.53 & 0.057 \\
\hline Planning & 9.9 & 5.0 & 9.6 & 10.5 & 3.82 & 0.282 \\
\hline Suicidal attempt & 6.9 & 5.0 & 3.6 & 2.6 & 4.14 & 0.247 \\
\hline Anxiety (\%) & 85.9 & 48.8 & 91.6 & 86.8 & 135.03 & $0.000^{*}$ \\
\hline $\begin{array}{l}\text { Loss of sleep because of worries } \\
\text { (last year, \%) }\end{array}$ & 57.8 & 48.4 & 48.0 & 35.1 & 12.74 & $0.005^{*}$ \\
\hline
\end{tabular}




\begin{tabular}{lcccccc}
\hline & \multicolumn{2}{c}{ Université de Saint-Boniface } & \multicolumn{2}{c}{ Brandon University } & \\
\cline { 2 - 5 } \multicolumn{1}{c}{ Variable } & $\begin{array}{c}\text { Domestic } \\
\text { students } \\
n=467\end{array}$ & $\begin{array}{c}\text { International } \\
\text { students } \\
n=172\end{array}$ & $\begin{array}{c}\text { Domestic } \\
\text { students } \\
n=255\end{array}$ & $\begin{array}{c}\text { International } \\
\text { students } \\
n=38\end{array}$ & $\begin{array}{c}\text { Observed } \\
\text { statistic }^{1}\end{array}$ & $p$ value \\
\hline $\begin{array}{l}\text { Used laxative to control weight } \\
\text { (last year, \%) }\end{array}$ & 4.2 & 16.3 & 6.0 & 10.5 & 27.20 & $0.000^{*}$ \\
\hline $\begin{array}{l}\text { Exercised excessively (last year, } \\
\%)\end{array}$ & 31.6 & 46.1 & 39.8 & 34.2 & 11.82 & $0.008^{*}$ \\
\hline
\end{tabular}

Psychological characteristics

\begin{tabular}{|c|c|c|c|c|c|c|}
\hline \multicolumn{7}{|l|}{ Satisfaction with life (\%) } \\
\hline Satisfied & $57 . .6$ & 66.1 & 62.9 & 60.6 & \multirow{3}{*}{10.06} & \multirow{3}{*}{0.122} \\
\hline Uncertain & 20.3 & 11.5 & 17.1 & 26.3 & & \\
\hline Dissatisfied & 22.1 & 22.4 & 19.9 & 13.2 & & \\
\hline \multicolumn{7}{|l|}{ Self-reported self-esteem (\%) } \\
\hline Positive & 51.0 & 80.5 & 52.8 & 68.4 & \multirow{3}{*}{58.89} & \multirow{3}{*}{$0.000^{*}$} \\
\hline Uncertain & 15.7 & 9.1 & 15.5 & 26.3 & & \\
\hline Negative & 33.3 & 10.4 & 31.7 & 5.3 & & \\
\hline Self-esteem score (mean) $)^{2, a, c, d}$ & 28.3 & 31.4 & 29.15 & 31.37 & 11.34 & $0.000^{*}$ \\
\hline \multicolumn{7}{|l|}{$\begin{array}{l}\text { Satisfaction with body image or } \\
\text { size (last month, \%) }\end{array}$} \\
\hline Never & 15.3 & 4.3 & 17.5 & 15.8 & \multirow{4}{*}{90.61} & \multirow{4}{*}{$0.000^{*}$} \\
\hline Sometimes & 38.8 & 31.5 & 40.1 & 28.9 & & \\
\hline Most of the time & 36.1 & 24.7 & 29.8 & 28.9 & & \\
\hline Always & 9.8 & 39.5 & 12.7 & 26.3 & & \\
\hline
\end{tabular}

Notes: Data are reported in percentages; $p$ value calculated using Chi-square or Exact tests except for mental health and self-esteem scores.

1: $\chi^{2}$ de Pearson or $\mathrm{F}$ statistic.

2: $p$ value calculated using Anova test.

Pairwise comparison for Anova test: a: Domestic USB vs. International USB; b: Domestic USB vs. Domestic BU; c: Domestic USB vs. International BU; d: International USB vs. Domestic BU; e: International USB vs. International BU; f: Domestic BU vs. International BU.

* Statistical significance at $5.0 \%$. 


\section{Table 4}

Mental health indicators and psychological characteristics, adjusted for age and gender

\begin{tabular}{|c|c|c|c|c|}
\hline \multirow{2}{*}{ Variable } & \multicolumn{2}{|c|}{ International vs. domestic } & \multicolumn{2}{|c|}{ USB vs. Brandon } \\
\hline & AOR $(95 \% \mathrm{Cl})$ & $p$ value $\dagger$ & AOR $(95 \% \mathrm{Cl})$ & $p$ value $\dagger$ \\
\hline \multicolumn{5}{|c|}{ Mental health indicators } \\
\hline $\begin{array}{l}\text { Self-reported positive } \\
\text { mental health }^{1}\end{array}$ & $3.44(2.3 ; 5.0)$ & $0.000^{*}$ & $0.97(0.72 ; 1.3)$ & 0.830 \\
\hline $\begin{array}{l}\text { Flourishing mental } \\
\text { health }^{2}\end{array}$ & $1.95(1.32 ; 2.88)$ & $0.001^{*}$ & $1.41(1.04 ; 1.92)$ & $0.028^{*}$ \\
\hline Depression $^{3}$ & $1.6(1.11 ; 2.29)$ & $0.011^{*}$ & $1.51(1.12 ; 2.04)$ & $0.007^{*}$ \\
\hline Anxiety ${ }^{4}$ & $0.24(0.16 ; 0.36)$ & $0.000^{*}$ & $0.39(0.24 ; 0.63)$ & $0.000^{*}$ \\
\hline Loss of sleep ${ }^{5}$ & $0.8(0.56 ; 1.14)$ & 0.218 & $1.45(1.08 ; 1.95)$ & $0.013^{*}$ \\
\hline \multicolumn{5}{|c|}{ Psychological characteristics } \\
\hline Life satisfaction ${ }^{6}$ & $1.54(1.11 ; 1.13)$ & $0.009^{*}$ & $1.08(0.83 ; 1.4)$ & 0.541 \\
\hline Self-esteem ${ }^{7}$ & $2.63(1.79 ; 4.0)$ & $0.000^{*}$ & $1.05(0.78 ; 1.39)$ & 0.746 \\
\hline Positive body image ${ }^{8}$ & $2.11(1.53 ; 2.92)$ & $0.000^{*}$ & $1.15(0.88 ; 1.5)$ & 0.297 \\
\hline
\end{tabular}

AOR: Adjusted Odds Ratio, adjusted for age and gender.

Cl: confidence interval.

$\dagger$ : value $p$ for binary or ordinal logistic regression adjusted for age and gender.

* Statistical significance at $5.0 \%$

1: Goodness of fit of multivariate ordinal logistic regression: Pearson $\left(\chi^{2}=253.48 ; \mathrm{df}=298 ; p=0.971\right)$ \& Deviance $\left(\chi^{2}=256.57 ; \mathrm{df}=298 ; p=\right.$ $0.960)$.

2: Goodness of fit of multivariate ordinal logistic regression: Pearson $\left(\chi^{2}=278.17 ; d f=284 ; p=0.566\right) \&$ Deviance $\left(\chi^{2}=264.37 ; d f=284 ; p=\right.$ 0.793).

3: Hosmer-Lemeshow goodness-of-fit test showed that the multivariate logistic binary logistic model was appropriate $\left(\chi^{2}=7.94 ; \mathrm{df}=8 ; p=\right.$ 0.439).

4: Hosmer-Lemeshow goodness-of-fit test showed that the multivariate logistic binary logistic model was appropriate $\left(\chi^{2}=6.42 ; \mathrm{df}=7 ; p=\right.$ $0.491)$.

5: Hosmer-Lemeshow goodness-of-fit test showed that the multivariate logistic binary logistic model was appropriate $\left(\chi^{2}=12.22 ; \mathrm{df}=8 ; p=\right.$ $0.142)$.

6: Goodness of fit of multivariate ordinal logistic regression: Pearson $\left(\chi^{2}=283.21 ; \mathrm{df}=298 ; p=0.722\right) \&$ Deviance $\left(\chi^{2}=302.71 ; \mathrm{df}=298 ; p=\right.$ $0.913)$.

7: Goodness of fit of multivariate ordinal logistic regression: Pearson $\left(\chi^{2}=314.71 ; \mathrm{df}=296 ; p=0.218\right)$ \& Deviance $\left(\chi^{2}=267.51 ; \mathrm{df}=296 ; p=\right.$ $0.881)$.

8: Goodness of fit of multivariate ordinal logistic regression: Pearson $\left(\chi^{2}=530.55 ; \mathrm{df}=449 ; p=0.005\right) \&$ Deviance $\left(\chi^{2}=488.68 ; \mathrm{df}=449 ; p=\right.$ 0.095).

\section{Coping Strategies and Supports}

As shown in Table 5, IS were less likely to report coping skills than domestic students, particularly at USB. These management strategies included individual or group counselling, medication (prescription and self-medication), student accommodation services, healthy living habits, and meditation or mindfulness. Coping strategies most often reported by IS were healthy living and meditation or mindfulness. 


\section{Table 5}

Coping strategies for anxiety for domestic and international students, by university

\begin{tabular}{|c|c|c|c|c|c|c|}
\hline \multirow[b]{2}{*}{ Variable } & \multicolumn{2}{|c|}{ Université de Saint-Boniface } & \multicolumn{2}{|c|}{ Brandon University } & \multirow[b]{2}{*}{$\begin{array}{l}\text { Observed } \\
\text { statistic }^{1}\end{array}$} & \multirow[b]{2}{*}{$p$ value } \\
\hline & $\begin{array}{c}\text { Domestic } \\
\text { students } \\
n=467\end{array}$ & $\begin{array}{c}\text { International } \\
\text { students } \\
n=172 \\
\end{array}$ & $\begin{array}{c}\text { Domestic } \\
\text { students } \\
n=255\end{array}$ & $\begin{array}{c}\text { International } \\
\text { students } \\
n=38\end{array}$ & & \\
\hline \multicolumn{7}{|c|}{ Coping strategies } \\
\hline $\begin{array}{l}\text { Individual or group therapy or } \\
\text { counselling }\end{array}$ & 15.9 & 3.9 & 14.1 & 7.9 & 15.94 & $0.000^{*}$ \\
\hline Prescription medication & 11.3 & 3.9 & 16.1 & 5.3 & 15.90 & $0.001^{*}$ \\
\hline $\begin{array}{l}\text { Self-medication (including } \\
\text { cannabis) }\end{array}$ & 14.5 & 5.2 & 19.0 & 5.3 & 17.86 & $0.000^{*}$ \\
\hline Student accommodation services & 6.0 & 1.3 & 3.2 & 5.3 & 7.17 & 0.050 \\
\hline Healthy living & 63.7 & 22.6 & 56.9 & 47.4 & 79.55 & $0.000^{*}$ \\
\hline $\begin{array}{l}\text { Meditation or mindfulness } \\
\text { techniques }\end{array}$ & 35.8 & 16.10 & 38.7 & 34.2 & 25.14 & $0.000^{*}$ \\
\hline \multicolumn{7}{|c|}{ Someone to talk to } \\
\hline $\begin{array}{l}\text { Don't feel the need, therefore no } \\
\text { one }\end{array}$ & 10.9 & 21.5 & 12.0 & 13.2 & 11.446 & $0.014^{*}$ \\
\hline No one & 9.7 & 14.1 & 6.0 & 0.0 & 11.594 & $0.007^{*}$ \\
\hline Spouse/partner & 44.3 & 28.2 & 49.8 & 31.6 & 20.415 & $0.000^{*}$ \\
\hline One of my parents & 63.6 & 35.6 & 57.8 & 44.7 & 37.828 & $0.000^{*}$ \\
\hline Brother or sister & 40.5 & 38.3 & 43.8 & 47.4 & 1.922 & 0.586 \\
\hline Friend & 78.5 & 51.0 & 78.1 & 63.2 & 48.606 & $0.000^{*}$ \\
\hline $\begin{array}{l}\text { Counsellor or therapist on } \\
\text { campus }\end{array}$ & 5.0 & 5.4 & 5.2 & 5.3 & 0.041 & 0.994 \\
\hline Health professional & 11.5 & 5.4 & 11.2 & 0.0 & 9.39 & $0.014^{*}$ \\
\hline Spiritual counsellor & 4.3 & 4.0 & 3.6 & 7.9 & 1.55 & 0.606 \\
\hline
\end{tabular}

Notes: Data are reported in percentages; $p$ value calculated using Chi-square or Exact tests.

1: $\chi^{2}$ de Pearson or F statistic.

* Statistical significance at $5.0 \%$.

When asked who they confided in when the need arose, a significantly higher proportion of USB IS reported not feeling the need to confide in someone (21.5\%) compared to domestic students (10.9\%). USB and BU IS were less likely to confide in a spouse/partner, a parent, friend, or a healthcare professional than their domestic counterparts, although all groups similarly reported con- fiding in a counsellor or therapist on campus, or in a sibling. Few confided in spiritual counsellors.

\section{Discussion}

Present findings contribute valuable insight contrasting international and domestic post-secondary student men- 
tal health statuses, risk-taking behaviours, coping strategies, and support access whilst studying in Manitoba in either of Canada's official languages. Overall, IS were more likely to report excellent general health and flourishing mental health than domestic students, as well as greater life satisfaction, self-esteem, and positive body image. However, IS generally reported engaging in fewer coping mechanisms and confiding in others about their worries less frequently compared to domestic students. Few reported seeking formal counselling or therapy from healthcare professionals. Of interest, international and domestic students on the French-language campus were more likely to report depressive symptomology, whereas respondents on the English-language campus were more likely to report anxiety.

Present findings pertaining to mental health status may seem surprising given that the literature has generally established that IS face greater mental health difficulties and exhibit more mental health-related risk-taking behaviours than their domestic counterparts (Kim et al., 2019; Shadowen et al., 2019). Our findings may, in part, be explained by our IS sociodemographic profile: most were male, older, and more likely to report religious practice. These characteristics may have influenced results and contributed to higher mental health scores (Kim et al., 2019). Furthermore, the present study's unique context -that of African IS on smaller Canadian campuses-contrasts with research previously undertaken in larger metropolitan institutions (Robinson et al., 2016; Thomson \& Esses, 2016) and among Asian IS (Huang \& Mussap, 2018; Thomson \& Esses, 2016; Zhang \& Goodson, 2011b). As cultures present in African countries may be more distant from Western ethos than many Asian cultures (Zhang \& Goodson, 2011a), African IS may perceive mental health differently, and as such, may present with distinct MHL and symptomology. Further analysis is needed to determine correlations between specific sociodemographic factors and mental health status of IS. A qualitative study, seeking to better understand these findings, is currently underway.

\section{Mental Health Profiles of Ethnolinguistically Diverse Populations}

To our knowledge, this study is the first to compare mental health status and coping strategies of English and French-speaking IS studying in Canada's bilingual set- ting. French-speaking IS reported higher mental health and self-esteem scores, although they were more likely to report depression and loss of sleep compared to English-speaking IS. As demonstrated by Gallais et al. (2020), IS enrolled in French-language post-secondary institutions in Quebec (where French is the language of majority), New Brunswick, and Prince-Edward-Island (provinces with French-language minority populations) fared better than French-speaking domestic students in Quebec (Gallais et al., 2020; Martineau et al., 2017). Similar to our findings, Gallais et al. (2020) also found French-speaking IS to be more likely to report symptoms of depression than anxiety. This could be linked to diverging MHL depending on students' culture of origin, or to ethnolinguistic identity, which has been identified as a mediator of mental health (Usborne \& Taylor, 2010). Interestingly, English-speaking IS obtained higher ethnolinguistic identity scores than French-speaking IS or domestic students. As shown by Tobler et al. (2013), a strong sense of belonging to one's ethnolinguistic group may be associated with perceived discrimination based on ethnicity, thus having a negative impact on mental health. Further research is needed to gain insight into the impact of ethnolinguistic and cultural identity on IS mental health in both Canadian linguistic contexts.

Language proficiency may also influence the acculturation and well-being of IS. Upon entering a bilingual country, IS may reasonably expect to live, work, and benefit from services in either official language. For French-speaking IS, who infrequently speak English proficiently, an altogether different reality awaits them: gainful employment, social interactions, and healthcare services in French are often difficult to access in official language minority communities such as in Winnipeg (de Moissac \& Bowen, 2017). Belonging to an intersection of multiple minorities (Mianda, 2018) and experiencing significant language barriers, French-speaking immigrants in Winnipeg may face greater obstacles in accessing information and care related to their general or mental health (Archambault et al., 2020). Efforts should aim to mitigate language barriers by providing adequate opportunities to learn English, whether through course work, volunteer opportunities, or twinning programs between international and domestic students, as such programs improve immigrant settlement and overall mental health (Chadwick \& Collins, 2015). General and mental health care and supports should be actively offered in the offi- 
cial language of choice, to promote safe and appropriate care (Bowen, 2015). Ultimately, actively involving IS in educational, social, and community settings where they can comfortably communicate in either official language, will empower IS to better integrate into the Canadian society.

\section{Religious Practice}

Religious practice, more prevalent among IS than domestic students, may have a mitigative effect on acculturative stress and academic performance, as it provides an internal source of hope and guidance, supports resilience by enhancing physical and mental well-being, and protects against depressive symptomology brought on by perceptions of discrimination (Fernandez \& Loukas, 2014; Philip et al., 2019). Students may find familiarity, structure, emotional and psychological refuge, as well as support, in their communities of faith and worship (Chai et al., 2012; Gardner et al., 2014). It is surprising that few IS in the present study sought support from faith leaders, as it is common for faith healers to be the first point of contact when physical or mental health difficulties arise (Graham \& White, 2015); this may, however, be explained by a paucity of faith leaders proficient in students' native language in proximity to campus housing. Facilitating contact with such faith leaders and providing a place of worship on campus inclusive to all faith traditions may be beneficial, as these provide valuable opportunities for religious practice and meditation. Such a space is available to all students at USB and may in part support our finding that USB IS reported healthier mental health outcomes.

\section{Culturally Adapted Supports and Services}

IS generally reported engaging in fewer coping mechanisms and confiding in others about their worries less frequently compared to domestic students. Help-seeking behaviours were particularly limited among USB IS, which may be partly explained by the local paucity of services available in French. IS may, however, possess unique culturally based coping mechanisms that differ from usual Western understandings and strategies. Fewer help-seeking behaviours may also be linked to increased stigma associated with mental health (Levesque
\& Rocque, 2019; Smith \& Khawaja, 2011), which may result in lesser ease, confidence, or acceptability in admitting to symptoms and subsequently seeking assistance (Arthur et al., 2010). MHL may also be underdeveloped in IS (Beks et al., 2019; Clough et al., 2019), who may not be familiar with words like "stress" or "depression" (Gallais et al., 2020; Levesque \& Rocque, 2019). It is, however, important to note that few studies have explored MHL among citizens of sub-Saharan countries, and many have employed tools developed for Western perceptions and cultures, which may hinder their validity (Atilola, 2015; Gallais et al., 2020). Others have found that, although some immigrants from sub-Saharan countries did adhere to similar mental health understandings as in Western societies, important variabilities exist and must be taken into account (Levesque \& Rocque, 2015, 2019). To be effective, healthcare providers must remain actively conscious of cultural norms to ensure that communication and service provision are culturally-congruent (Beks et al., 2019; Xu et al., 2018).

As suggested by our findings, IS who have recently entered Canada exhibit a more positive outlook and healthier psychological characteristics, favouring positive mental health outcomes. This may be linked to the newcomer effect: enthusiasm and novelty felt by IS as they enter a new country of residence (Conchas et al., 2012). As this excitement wears off, however, and the reality of their situation sets in, more insidious symptoms may arise, for which they may be ill-equipped. Ongoing monitoring of IS' mental health status and evaluation of the efficaciousness of available supports throughout their academic journey is vital (Gallais et al., 2020). IS are a valuable presence in the Canadian post-secondary landscape; support services are vital not only for their academic and personal fulfillment, but also for their retention and integration into the Canadian society. Canadian campuses should provide culturally-adapted support services, tailored to this heterogeneous international clientele.

\section{Limitations}

This quantitative study, in contrast to qualitative studies often conducted to gain insight into IS' experiences, allows for conclusions based on statistical evidence. It should be noted, however, that BU's sample is small, despite efforts to recruit adequate representation of all student subpopulations. As not all faculty agreed to for- 
feit class time for data collection, many students did not participate; data pertaining to this cohort should be considered with caution. This study was conducted in two mid-sized universities in Manitoba; findings may only be generalizable to similar-sized universities and in provinces with comparable conditions related to recruitment and provision of services to IS, both on-campus and in the larger community. Study of diverse interprovincial contexts and frameworks may reveal significant differences in IS personal and academic health and success; interprovincial differences merit further study. As many studies on the well-being of IS have focused primarily on students from Asian countries, in contrast to the majority of our participants who were from African countries, our findings shed light on an under-researched demographic with unique cultural and linguistic qualities.

\section{Conclusion}

This quantitative study provides a descriptive snapshot of IS well-being while studying within two distinct linguistic realities in Manitoba, Canada. These students were found to exhibit stronger overall mental health and psychological characteristics than domestic students. Further research is needed to gain insight into protective factors leading to enhanced mental health status. Culturally congruent support systems are needed on Canadian campuses. These should aim to ensure that the heterogeneous beliefs, traditions, and worldviews of IS are valued and nurtured on our campuses.

\section{References}

Abraído-Lanza, A. F., White, K., \& Vásquez, E. (2004). Immigrant populations and health. In N. Anderson (Ed.), Encyclopedia of health and behavior (Vol. 2, pp. 533-537). Sage.

Acharya, L., Jin, L., \& Collins, W. (2018). College life is stressful today - Emerging stressors and depressive symptoms in college students. Journal of American College Health, 66(7), 655-664. https://doi.org/10.10 80/07448481.2018.1451869

American College Health Association. (2019). National college health assessment undergraduate student reference group executive summary (National College Health Assessment, pp. 1-22) [Executive
Summary]. American College Health Association. https://www.acha.org/NCHA/ACHA-NCHA Datal Publications and Reports/NCHA/Data/Reports ACHA-NCHAll..aspx

Anderson, T. (2015). Seeking internationalization: The state of Canadian higher education. Canadian Journal of Higher Education, 45(4), 166-187. https:// doi.org/10.47678/cjhe.v45i4.184690

Archambault, H., de Moissac, D., Levesque, A., Kinkumba, B., Gueye, N. R., Tempier, R., \& Alimezelli, H. T. (2020). Expérience d'immigration de Francophones en contexte linguistique minoritaire au Canada: défis et répercussions. Manuscript submitted for publication.

Arnett, J. J. (2005). The developmental context of substance use in emerging adulthood. Journal of Drug Issues, 35(2), 235-254. https://doi. org/10.1177/002204260503500202

Arthur, C. M., Hickling, F. W., Robertson-Hickling, H., Haynes-Robinson, T., Abel, W., \& Whitley, R. (2010). "Mad, Sick, Head Nuh Good": Mental illness stigma in Jamaican communities. Transcultural Psychiatry, 47(2), 252-275. https://doi. org/10.1177/1363461510368912

Atilola, O. (2015). Level of community mental health literacy in sub-Saharan Africa: Current studies are limited in number, scope, spread, and cognizance of cultural nuances. Nordic Journal of Psychiatry, 69(2), 93-101. https://doi.org/10.3109/08039488.20 $\underline{\underline{14.947319}}$

Bartram, B., \& Terano, M. (2011). Supporting international students in higher education: A comparative examination of approaches in the U.K. and U.S.A. Learning and Teaching, 4(2), 30-44. https://doi. org/10.3167/latiss.2011.040203

Beks, T. A., Cairns, S. L., Smygwaty, S., Miranda Osorio, O. A. L., \& Hill, S. J. (2019). Counsellor-in-residence: Evaluation of a residence-based initiative to promote student mental health. Canadian Journal of Higher Education, 48(2), 55-73. https://doi. org/10.7202/1057103ar

Berry, J. W. (2005). Acculturation: Living successfully in two cultures. International Journal of Intercultural 
Relations, 29(6), 697-712. https://doi.org/10.1016/j. ijintrel.2005.07.013

Bowen, S. (2015). The impact of language barriers on patient safety and quality of care (pp. 1-53). Société Santé en français. http://www.santefrancais.cal wp-content/uploads/2018/11/SSF-Bowen-S.-Language-Barriers-Study-1.pdf

Brailovskaia, J., \& Margraf, J. (2018). How to measure self-esteem with one item? Validation of the German single-item self-esteem scale (G-SISE). Current Psychology, 39, 2192-2202. https://doi.org/10.1007/ s12144-018-9911-X

Brisset, C., Leanza, Y., Rosenberg, E., Vissandjée, B., Kirmayer, L. J., Muckle, G., Xenocostas, S., \& Laforce, $H$. (2014). Language barriers in mental health care: A survey of primary care practitioners. Journal of Immigrant and Minority Health, 16(6), 1238-1246. https://doi.org/10.1007/s10903-013-9971-9

Calder, M. J., Richter, S., Mao, Y., Burns, K. K., Mogale, R. S., \& Danko, M. (2016). International students attending Canadian universities: Their experiences with housing, finances, and other issues. Canadian Journal of Higher Education, 46(2), 92-110. https:ll doi.org/10.47678/cjhe.v46i2.184585

Canadian Bureau for International Education. (2018a). International students in Canada. CBIE Research in Brief \#10. https://cbie.ca/what-we-do/research/ libraryl

Canadian Bureau for International Education. (2018b). The student's voice: National results of the 2018 CBIE International Student Survey. CBIE Research in Brief \#9. https://cbie.ca/what-we-do/research/ libraryl

Chadwick, K. A., \& Collins, P. A. (2015). Examining the relationship between social support availability, urban center size, and self-perceived mental health of recent immigrants to Canada: A mixed-methods analysis. Social Science \& Medicine, 128, 220-230. https://doi.org/10.1016/j.socscimed.2015.01.036

Chai, P. P. M., Krägeloh, C. U., Shepherd, D., \& Billington, R. (2012). Stress and quality of life in international and domestic university students: Cultural differences in the use of religious coping. Mental
Health, Religion \& Culture, 15(3), 265-277. https:// doi.org/10.1080/13674676.2011.571665

Cleary, M., Walter, G., \& Jackson, D. (2011). “Not always smooth sailing": Mental health issues associated with the transition from high school to college. Issues in Mental Health Nursing, 32(4), 250-254. https://doi.org/10.3109/01612840.2010.548906

Clough, B. A., Nazareth, S. M., Day, J. J., \& Casey, L. M. (2019). A comparison of mental health literacy, attitudes, and help-seeking intentions among domestic and international tertiary students. British Journal of Guidance \& Counselling, 47(1), 123-135. https:ll doi.org/10.1080/03069885.2018.1459473

Conchas, G. Q., Oseguera, L., \& Vigil, J. D. (2012). Acculturation and school success: Understanding the variability of Mexican American youth adaptation across urban and suburban contexts. The Urban Review, 44(4), 401-422. https://doi.org/10.1007/ s11256-012-0197-2

Crockett, L. J., Iturbide, M. I., Torres Stone, R. A., McGinley, M., Raffaelli, M., \& Carlo, G. (2007). Acculturative stress, social support, and coping: Relations to psychological adjustment among Mexican American college students. Cultural Diversity and Ethnic Minority Psychology, 13(4), 347-355. https:/l doi.org/10.1037/1099-9809.13.4.347

Dawson, M., \& Pooley, J. A. (2013). Resilience: The role of optimism, perceived parental autonomy support and perceived social support in first year university students. Journal of Education and Training Studies, 1(2), 38-49. https://doi.org/10.11114/jets.v1i2.137

de Moissac, D., \& Bowen, S. (2017). Impact of language barriers on access to healthcare for official language minority Francophones in Canada. Healthcare Management Forum, 30(4), 207-212. https://doi. org/10.1177/0840470417706378

de Moissac, D., Gueye, N. R., \& Rocque, R. (2019). My health, your health, our health: A study on mental health and risk-taking behaviors of young adults on five university campuses in the heart of Canada ( $\mathrm{p}$. 194). Université de Saint-Boniface. https://ustboniface.ca/ddemoissac/file/Mental-Health-Englishv1.1.pdf 
Economic Development Brandon. (2020). Cost of living. Economic Development Brandon. http://economicdevelopmentbrandon.com/cost-of-living

Economic Development Winnipeg. (2020). Cost of living. Economic Development Winnipeg. https://www.economicdevelopmentwinnipeg.com/choose-winnipeg/ live-here/cost-of-living

Eisenberg, D., Downs, M. F., Golberstein, E., \& Zivin, K. (2009). Stigma and help seeking for mental health among college students. Medical Care Research and Review, 66(5), 522-541. https://doi. org/10.1177/1077558709335173

Farrell, M., \& Langrehr, K. J. (2017). Stress, social support, and psychosocial functioning of ethnically diverse students. Journal of College Counseling, 20(3), 208-223. https://doi.org/10.1002/jocc.12070

Fernandez, A., \& Loukas, A. (2014). Acculturation and religious coping as moderators of the association between discrimination and depressive symptoms among Mexican-American vocational students. Journal of Immigrant and Minority Health, 16(6), 1290-1293. https://doi.org/10.1007/s10903-0139952-z

Forbes-Mewett, H., \& Sawyer, A.-M. (2016). International students and mental health. Journal of International Students, 6(3), 661-677. https://uml.idm.oclc.org/ login?url=https://www-proquest-com.uml.idm.oclc. org/docview/1826523975?accountid=14569

Fritz, M. V., Chin, D., \& DeMarinis, V. (2008). Stressors, anxiety, acculturation and adjustment among international and North American students. International Journal of Intercultural Relations, 32(3), 244-259. https://doi.org/10.1016/j.jijintrel.2008.01.001

Gallais, B., Nguema, N. B. B., Parent, S. J., \& Turcotte, A. (2020). Enjeux et défis de l'adaptation, de l'intégration et de la réussite scolaire des étudiants internationaux dans les cégeps et les collèges francophones du Canada (pp. 1-48). Centre d'Étude des Conditions de vie et des Besoins de la population (ÉCOBES) - Recherche et transfert. https:/l eduq.info/xmlui/bitstream/handle/11515/37950/enjeux-defis-adaptation-etudiants-internationaux-ecobes-2020.pdf?sequence=2\&isAllowed $=y$
Gardner, T. M., Krägeloh, C. U., \& Henning, M. A. (2014). Religious coping, stress, and quality of life of Muslim university students in New Zealand. Mental Health, Religion \& Culture, 17(4), 327-338. https:Il doi.org/10.1080/13674676.2013.804044

Gloria, C. T., \& Steinhardt, M. A. (2016). Relationships among positive emotions, coping, resilience and mental health: Positive emotions, resilience and health. Stress and Health, 32(2), 145-156. https:// doi.org/10.1002/smi.2589

Graham, J. M., \& White, D. (2015). Muslim nursing student beliefs about possession states: An exploratory survey of beliefs and causal attributions. The Journal of Educational Thought (JET) / Revue de La Pensée Éducative, 48(1/2), 43-60. https://doi. org/10.11575/jet.v48i1\%20\&\%202.44217

Guo, Y., \& Guo, S. (2017). Internationalization of Canadian higher education: Discrepancies between policies and international student experiences. Studies in Higher Education, 42(5), 851-868. https://doi.org/ 10.1080/03075079.2017.1293874

Huang, S. L., \& Mussap, A. J. (2018). Maladaptive perfectionism, acculturative stress and depression in Asian international university students. Journal of Psychologists and Counsellors in Schools, 28(2), 185-196. https://doi.org/10.1017/jgc.2016.18

Keyes, C. L. M. (2002). The mental health continuum: From languishing to flourishing in life. Journal of Health and Social Behavior, 43(2), 207. https://doi. org/10.2307/3090197

Keyes, C. L. M. (2009). Atlanta: Brief description of the mental health continuum short form (MHC-SF). http://www. aacu.org/sites/default/files/MHC-SFEnglish.pdf

Kim, Y. K., Maleku, A., Lemieux, C. M., Du, X., \& Chen, Z. (2019). Behavioral health risk and resilience among international students in the United States: A study of socio-demographic differences. Journal of International Students, 9(1), 282-305. https://doi. org/10.32674/jis.v9i1.264

Kirmayer, L. J., Narasiah, L., Munoz, M., Rashid, M., Ryder, A. G., Guzder, J., Hassan, G., Rousseau, C., \& Pottie, K. (2011). Common mental health problems 
in immigrants and refugees: General approach in primary care. Canadian Medical Association Journal, 183(12), E959-E967. https://doi.org/10.1503/ cmaj.090292

Kovess-Masfety, V., Leray, E., Denis, L., Husky, M., Pitrou, I., \& Bodeau-Livinec, F. (2016). Mental health of college students and their non-college-attending peers: Results from a large French cross-sectional survey. BMC Psychology, 4(1), 20. https://doi. org/10.1186/s40359-016-0124-5

Kruisselbrink Flatt, A. (2013). A suffering generation: Six factors contributing to the mental health crisis in North American higher education. College Quarterly, 16(1). https://uml.idm.oclc.org/login?url=https://www-proquest-com.uml.idm.oclc.org/ docview/1651852277?accountid=14569

Levesque, A., \& Rocque, R. (2015). Représentations culturelles des troubles de santé mentale chez les immigrants et réfugiés de l'Afrique francophone subsaharienne au Canada. Alterstice: Revue Internationale de La Recherche Interculturelle, 5(1), 69-82. https://www.journal.psy.ulaval.ca/ojs/index.php/ARlRl/article/view/Levesque Alterstice5\%281\%29

Levesque, A., \& Rocque, R. (2019). Définitions et conséquences des troubles de santé mentale: Une analyse qualitative des représentations des migrants et migrantes de l'Afrique subsaharienne à Winnipeg. Canadian Journal of Community Mental Health, 38(2), 19-34. https://doi.org/10.7870/cjcmh-2019-004

Lokkesmoe, K. J., Kuchinke, K. P., \& Ardichvili, A. (2016). Developing cross-cultural awareness through foreign immersion programs: Implications of university study abroad research for global competency development. European Journal of Training and Development, 40(3), 155-170. https://doi. org/10.1108/EJTD-07-2014-0048

Martineau, M., Beauchamp, G., \& Marcotte, D. (2017). Efficacité des interventions en prévention et en promotion de la santé mentale dans les établissements d'enseignement postsecondaire. Santé Mentale au Québec, 42(1), 165-182. https://doi. org/10.7202/1040249ar

Martirosyan, N. M., Bustamante, R., \& Saxon, D. P.
(2019). Academic and social support services for international students. Journal of International Students, 9(1), 172-191. https://doi.org/10.32674/ jis.v9i1.275

Mesidor, J. K., \& Sly, K. F. (2016). Factors that contribute to the adjustment of international students. Journal of International Students, 6(1), 262-282. https://uml. idm.oclc.org/login?url=https://www-proquest-com. uml.idm.oclc.org/docview/1773215927?accountid $=14569$

Mianda, G. (2018). Genre, langue et race: L'expérience d'une triple marginalité dans l'intégration des immigrants francophones originaires de l'Afrique subsaharienne à Toronto, Canada. Francophonies d'Amérique, 46-47, 27-49. https://doi-org.uml.idm. oclc.org/10.7202/1064886ar

Nelson, L. J., \& Padilla-Walker, L. M. (2013). Flourishing and floundering in emerging adult college students. Emerging Adulthood, 1(1), 67-78. https://doi. org/10.1177/2167696812470938

Onabule, A. I., \& Boes, S. R. (2013). International students' likelihood to seek counseling while studying abroad. Journal of International Students, 3(1), 52-59. https://uml.idm.oclc.org/login?url=https://www-proquest-com.uml.idm.oclc.org/ docview/1697497976? accountid=14569

Owens, M., Stevenson, J., Hadwin, J. A., \& Norgate, R. (2012). Anxiety and depression in academic performance: An exploration of the mediating factors of worry and working memory. School Psychology International, 33(4), 433-449. https://doi. org/ $/ 10.1177 / 0143034311427433$

Philip, S., Neuer Colburn, A. A., Underwood, L., \& Bayne, H. (2019). The impact of religion/spirituality on acculturative stress among international students. Journal of College Counseling, 22(1), 27-40. https://doi.org/10.1002/jocc.12112

Phinney, J. S. (1992). The multigroup ethnic identity measure: A new scale for use with diverse groups. Journal of Adolescent Research, 7(2), 156-176. https://doi.org/10.1177/074355489272003

Ponterotto, J. G., Gretchen, D., Utsey, S. O., Stracuzzi, T., \& Saya, R. (2003). The multigroup ethnic 
identity measure (MEIM): Psychometric review and further validity testing. Educational and Psychological Measurement, 63(3), 502-515. https://doi. org/10.1177/0013164403063003010

Prieto-Welch, S. L. (2016). International student mental health. New Directions for Student Services, 2016(156), 53-63. https://doi.org/10.1002/ss.20191

Province of Manitoba. (2020). Live in Manitoba I Manitoba immigration and economic opportunities. Province of Manitoba. https://www.immigratemanitoba.com/choose-manitoba/live-in-manitobal

Rafal, G., Gatto, A., \& DeBate, R. (2018). Mental health literacy, stigma, and help-seeking behaviors among male college students. Journal of American College Health, 66(4), 284-291. https://doi.org/10.1080/0744 8481.2018 .1434780

Roberts, R. E., Phinney, J. S., Masse, L. C., Chen, Y. R., Roberts, C. R., \& Romero, A. (1999). The structure of ethnic identity of young adolescents from diverse ethnocultural groups. The Journal of Early Adolescence, 19(3), 301-322. https://doi. org/10.1177/0272431699019003001

Robertson, L. H., Holleran, K., \& Samuels, M. (2015). Tailoring university counselling services to Aboriginal and international students: Lessons from Native and international student centres at a Canadian university. Canadian Journal of Higher Education, 45(1), 122-135. https://uml.idm.oclc.org/login?url=https://www-proquest-com.uml.idm.oclc.org/ docview/1697486187?accountid=14569

Robinson, A. M., Jubenville, T. M., Renny, K., \& Cairns, S. L. (2016). Academic and mental health needs of students on a Canadian campus. Canadian Journal of Counselling and Psychotherapy, 50(2), 108-123. http://ovidsp.ovid.com/ ovidweb.cgi? T=JS\&PAGE=reference\&D=psyc13\&NEWS=N\&AN=2016-35579-002

Schwitzer, A. M., Moss, C. B., Pribesh, S. L., John, D. J. S., Burnett, D. D., Thompson, L. H., \& Foss, J. J. (2018). Students with mental health needs: College counseling experiences and academic success. Journal of College Student Development, 59(1), 3-20. https://doi.org/10.1353/csd.2018.0001
Shadowen, N. L., Williamson, A. A., Guerra, N. G., Ammigan, R., \& Drexler, M. L. (2019). Prevalence and correlates of depressive symptoms among international students: Implications for university support offices. Journal of International Students, 9(1), 129-149. https://doi.org/10.32674/jis.v9i1.277

Smith, C., Whiteside, B., Blanchard, S., \& Martin, C. (2013). International student support services at Ontario universities. Strategic Enrollment Management Quarterly, 1(1), 55-66. https://doi.org/10.1002/ $\underline{\text { sem3.20005 }}$

Smith, R. A., \& Khawaja, N. G. (2011). A review of the acculturation experiences of international students. International Journal of Intercultural Relations, 35(6), 699-713. https://doi.org/10.1016/j.jijintrel.2011.08.004

Statistics Canada. (2017, November 29). Winnipeg, cY [Census subdivision], Manitoba and Brandon [Census agglomeration], Manitoba (table). Census Profile. 2016 Census. Statistics Canada. https:/l www12.statcan.gc.ca/census-recensement/2016/dp$\mathrm{pd} /$ prof/index.cfm?Lang=E

Statistics Canada. (2018a). Canadian postsecondary enrolments and graduates, 2016/2017. The Daily, 11, 1-4.

Statistics Canada. (2018b). Postsecondary enrolments, by status of student in Canada, country of citizenship and gender. Statistics Canada. https://www150. statcan.gc.ca/t1/tbl1/en/tv.action?pid=3710008601

Statistics Canada. (2020). Canadian postsecondary enrolments and graduates, 2017/2018. The Daily, pp. 1-3.

Thomson, C., \& Esses, V. M. (2016). Helping the transition: Mentorship to support international students in Canada. Journal of International Students, 6(4), 873-886. https://uml.idm.oclc.org/login?url=https://www-proquest-com.uml.idm.oclc.org/ docview/1895979769?accountid=14569

Thomson, M. D., \& Hoffman-Goetz, L. (2009). Defining and measuring acculturation: A systematic review of public health studies with Hispanic populations in the United States. Social Science \& Medicine, 69(7), 983-991. https://doi.org/10.1016/j. socscimed.2009.05.011 
Tobler, A. L., Maldonado-Molina, M. M., Staras, S. A. S., O'Mara, R. J., Livingston, M. D., \& Komro, K. A. (2013). Perceived racial/ethnic discrimination, problem behaviors, and mental health among minority urban youth. Ethnicity \& Health, 18(4), 337-349. https://doi.org/10.1080/13557858.2012.730609

Usborne, E., \& Taylor, D. M. (2010). The role of cultural identity clarity for self-concept clarity, elf-esteem, and subjective well-being. Personality and Social Psychology Bulletin, 36(7), 883-897. https://doi. org/10.1177/0146167210372215

Xu, X., Li, X.-M., Zhang, J., \& Wang, W. (2018). Mental health-related stigma in China. Issues in Mental Health Nursing, 39(2), 126-134. https://doi.org/10.1 $\underline{080 / 01612840.2017 .1368749}$

Yang, N., Xu, Y., Chen, X., Yu, B., Yan, H., \& Li, S. (2018). Acculturative stress, poor mental health and condom-use intention among international students in China. Health Education Journal, 77(2), 142-155. https://doi.org/10.1177/0017896917739443

Zhang, J., \& Goodson, P. (2011a). Predictors of international students' psychosocial adjustment to life in the United States: A systematic review. International Journal of Intercultural Relations, 35(2), 139-162. https://doi.org/10.1016/j.jijintrel.2010.11.011

Zhang, J., \& Goodson, P. (2011b). Acculturation and psychosocial adjustment of Chinese international students: Examining mediation and moderation effects. International Journal of Intercultural Relations, 35(5), 614-627. https://doi.org/10.1016/j. ijiintrel.2010.11.004

\section{Contact Information}

Danielle de Moissac

ddemoissac@ustboniface.ca

\section{Notes}

1 International student support services commonly offered include: academic (language, writing and study skills, informational literacy), health (culturally-adapted counselling and access to healthcare), student advising (financial, career, childcare), social networking (social and cultural events, sports, clubs) and immigration assistance (Bartram \& Terano, 2011; Martirosyan et al., 2019).

2 Examples of supports tailored to international students on Canadian campuses include: academic, accommodation and living, employment, financial, health and wellness, immigration, social and cultural, and support for transitioning into the Canadian context (Smith et al., 2013).

3 Personalized support services offered to IS at USB include aids in settlement and integration into Manitoban academic life, and referrals to community support services available in French.

4 Ethics certificate from USB-ETH 201810 juillet, and BU-BUREC \#22315. 